\title{
Curcumin Protects against Renal Ischemia/Reperfusion Injury by Regulating Oxidative Stress and Inflammatory Response
}

\author{
Xiaoying Cui $\mathbb{D}^{1},{ }^{1}$ Lili Lin $\mathbb{D}^{2},{ }^{2}$ Xiaoling Sun $\mathbb{D}^{3},{ }^{3}$ Lin Wang $\mathbb{D}^{4},{ }^{4}$ and Rong Shen $\mathbb{D}^{5}$ \\ ${ }^{1}$ Department of Anesthesiology, The Cancer Hospital of the University of Chinese \\ Academy of Sciences (Zhejiang Cancer Hospital), Institute of Basic Medicine and Cancer (IBMC), Chinese Academy of Sciences, \\ Hangzhou 310022, China \\ ${ }^{2}$ Department of Anesthesiology, Qingdao Eighth People's Hospital, Qingdao 266100, China \\ ${ }^{3}$ Department of Acupuncture, Affiliated Qingdao Central Hospital, Qingdao University, Qingdao 266000, China \\ ${ }^{4}$ Department of Anesthesiology, Rizhao Central Hospital, Rizhao 276800, China \\ ${ }^{5}$ Department of Anesthesiology, Qingdao Women and Children's Hospital, Qingdao 266000, China
}

Correspondence should be addressed to Rong Shen; duyoudui3@163.com

Received 26 September 2021; Accepted 18 October 2021; Published 13 November 2021

Academic Editor: Muhammad Wasim Khan

Copyright (c) 2021 Xiaoying Cui et al. This is an open access article distributed under the Creative Commons Attribution License, which permits unrestricted use, distribution, and reproduction in any medium, provided the original work is properly cited.

\begin{abstract}
Objective. The aim of this study was to explore the pharmacological effects of curcumin on oxidative stress and inflammatory response of renal dysfunction induced by renal ischemia/reperfusion (RIRI). Methods. Fifty male SD rats (Sprague Dawley) were randomly divided into the sham group, RIRI group, and curcumin group (low, medium, and high). The RIRI model was established by clipping the left renal artery for $45 \mathrm{~min}$ and then reperfusion for $24 \mathrm{~h}$ and resection of the contralateral kidney. In the curcumin group, curcumin was intraperitoneally injected once a day for 3 consecutive days using different dosage regimens. The RIRI group was intraperitoneally administered with normal saline. Renal injury was evaluated by measuring the concentration of creatinine ( $\mathrm{Cr}$ ) and urea nitrogen (BUN) in serum. Oxidative stress was assessed by assessing the level of malondialdehyde (MDA), superoxide dismutase (SOD), catalase (CAT), glutathione peroxidase (GPx), glutathione (GSH), and iron reduction/antioxidant capacity (FRAP) in tissues. In addition, the protective effect of RIRI was investigated by measuring Paller scores, the level of serum inflammatory factors and caspase-3, and the number of apoptotic cells. Results. Ischemia/ reperfusion resulted in increased levels of $\mathrm{Cr}$ and BUN in serum and MDA in tissues and decreased levels of SOD, CAT, GPx, GSH, and FRAP. Curcumin pretreatment strikingly increased the level of SOD, CAT, GPx, GSH, IL-10, IFN- $\gamma$, and FRAP and significantly decreased MDA, Cr, BUN, IL-8, TNF- $\alpha$, IL-6, and myeloperoxidase (MPO) expressions in tissues. Conclusion. Curcumin can relieve the degree of renal injury and improve renal function in ischemia-reperfusion, which may be related to the fact that curcumin can increase SOD content in serum and reduce MDA and FRAP levels in the rat model.
\end{abstract}

\section{Introduction}

Renal ischemia-reperfusion injury (RIRI) is a common clinical pathological phenomenon, which is commonly seen in vascular surgery and kidney transplantation, and is one of the common causes of acute renal failure $[1,2]$. RIRI refers to that after the restoration of blood and oxygen supply to the renal organ after ischemia, the damage of the renal organ is aggravated or even irreversible, usually caused by an inflammatory cascade reaction, including reactive oxygen species (ROS), reactive nitrogen species (RNS), and cytokine, chemokine, and leukocyte activation [3, 4]. RIRI is a very complex pathological process, which mainly causes kidney damage through mitochondrial damage, inflammation, apoptosis, and oxidative stress. As an endocrine organ, the kidney is also a hyperperfusion organ, which is particularly sensitive to ischemia and reperfusion. When renal ischemia-reperfusion occurs, a large number of reactive oxygen species will be produced in the late stage of ischemia-reperfusion, which puts the kidney in a state of high oxidative stress and triggers a series of harmful cellular reactions, leading to inflammatory responses, cell apoptosis, 
and acute renal failure and even damage to other organs $[5,6]$.

Curcumin is a pigment extracted from turmeric, which is mainly distributed in tropical and subtropical areas such as India, China, and Southeast Asia. It is widely used as food pigment and has good safety in human body. Curcumin can reduce renal injury caused by renal ischemia-reperfusion by reducing oxidative stress response, upregulating APPL1 expression, inhibiting the Akt phosphorylation pathway, inhibiting activation of the INOS/NO/CGMP/PKG signaling pathway, inhibiting inflammatory cell infiltration, upregulating $\mathrm{HO}-1$, inhibiting NF- $\kappa \mathrm{B}$ activity, and reducing the production of vasoactive substances $[7,8]$. Curcumin has anti-inflammatory, antioxidant, antifibrosis, anticoagulation, and antitumor activities [9-12]. For instance, Nguyen-Ngo $\mathrm{C}$ et al. reported that curcumin markedly inhibited TNF-induced chemokines (CCL2-4, CXCL1, CXCL5, and CXCL8) expression, proinflammatory cytokines (IL- $1 \alpha$, IL- $1 \beta$, and IL- 6 ), and upregulated anti-inflammatory cytokines (IL-4 and IL-13) mRNA expression in visceral adipose tissue, human placenta, and subcutaneous adipose tissue [13]. Therefore, curcumin can be used as a new treatment method for renal ischemia-reperfusion (I/R). The purpose of this study was to investigate the protective effect of curcumin in antioxidant stress and inflammation in rats with IRI.

\section{Materials and Methods}

2.1. Animals. SPF male SD rats, weighing $240 \pm 20 \mathrm{~g}$, were provided by the Experimental Animal Center of the Nanjing University of Chinese Medicine. All experiments in this study were authorized by the Institutional Animal Care and Use Committee. SD rats were raised under standard SPF conditions, kept under controlled temperature conditions $\left(22-24^{\circ} \mathrm{C}\right)$, dark/light cycle for 12 hours, and allowed to eat and drink freely.

2.2. Drugs and Reagents. Curcumin (specification: $100 \mathrm{~g} /$ box, lot no.: 20181209) was purchased from Bio Basic, Canada. Superoxide dismutase (SOD) and malondialdehyde (MDA) detection kits were purchased from Sigma-Aldrich (St. Louis, MO, USA).

2.3. Establishment of Animal Models of Ischemia and Reperfusion. After ether anesthesia [14, 15], the body temperature was maintained at $37^{\circ} \mathrm{C}$, and abdominal incision through midline was performed with electrocautery to fully explore bilateral renal arteries and veins. At the same time, the renal arteries and veins were clamped bilaterally for 45 minutes. Once the ischemia time was reached, the clamps were released immediately [16]. At the end of the ischemia, the surgical area was sutured, and the rats were injected with curcumin or normal saline (NS) through a tail vein and free to get food and water. All tools used in this procedure are sterilized by autoclave or Deconex.

Throughout the procedure, a rectal probe was applied to measure the animal's temperature. A heating lamp and a heating plate were performed to keep the animal's body temperature within the range of $37 \pm 1^{\circ} \mathrm{C}$ [17].

2.4. Grouping and Administration. The rats were randomly divided into 5 groups (10 in each group): sham group (Sham), renal ischemia-reperfusion injury group (RIRI), RIRI-curcumin high-dose group $(60 \mathrm{mg} / \mathrm{kg})$, RIRI-curcumin medium-dose group $(30 \mathrm{mg} / \mathrm{kg})$, and RIRI-curcumin low-dose group $(15 \mathrm{mg} / \mathrm{kg})$. The sham group and RIRI group were given the same amount of normal saline in the tail vein, and in the curcumin group, curcumin was injected into the tail vein according to the dose, for a total of 5 days [18].

2.5. Renal Function Assessment. After $24 \mathrm{~h}$ of reperfusion, rats were anesthetized with $3 \%$ pentobarbital sodium $(50 \mathrm{mg} / \mathrm{kg}$ ) to obtain $2-5 \mathrm{~mL}$ of inferior venous blood. Then, the inferior venous blood was centrifuged at $2-8^{\circ} \mathrm{C}$ for $10 \mathrm{~min}$ at $3000 \mathrm{rpm}$. Then, the upper serum was collected and stored in a refrigerator at $-80^{\circ} \mathrm{C}$ for later experimental detection. The serum of rats was measured by an automatic analyzer (Technicon, RA-1000, USA) to evaluate renal function.

2.6. Renal Histomorphological Changes and Scoring. The renal tissues were fixed with $40 \mathrm{~g} / \mathrm{L}$ neutral paraformaldehyde solution for $24 \mathrm{~h}$, embedded in paraffin, stained with hematoxylin-eosin (H\&E), and observed under a microscope: 10 fields were randomly selected, 10 renal tubules were scored in each field, and the cortical medulla was divided in half. Renal tubules were scored according to Paller criteria: 100 renal tubules were scored with a total score of 1000 points. The higher the score was, the more serious the degree of renal tubule injury was.

2.7. Detection of Oxidative Stress Indicators. An appropriate amount of renal tissue was taken to prepare a $0.9 \%$ renal tissue homogenate using a tissue homogenizer and then centrifuged at $3000 \mathrm{rpm}$ at $4^{\circ} \mathrm{C}$ for $15 \mathrm{~min}$ to obtain the supernatant. MDA content was detected by the thiobarbituric acid method, and the content of SOD was detected by xanthine oxidase according to the instructions of the kit strictly. A total of $50 \mu \mathrm{L}$ tissue homogenate supernatant and FRAP concentration detection reagent were added to each test tube. FRAP levels were determined by measuring light absorption at $593 \mathrm{~nm}$. The levels of SOD, CAT, GPX, and GSH in serum were measured using ELISA kits as per manufacturer's instructions. All reagents were purchased from Sigma-Aldrich.

2.8. Detection of TNF- $\alpha, I L-6$, and IL-8 Levels. Total RNA was isolated from the entire kidney tissue using TRIzol (Japan, Takara) as per the manufacturer's instructions. Total RNA was then reversed using Advantage ${ }^{\circledR}$ RT-for-PCR Kit (Takara, Japan). Real-time PCR amplification was achieved using an ABI 7500 system (Massachusetts Thermoelectric, USA). Primers used for reverse transcription polymerase 
chain reaction (Invitrogen, Carlsbad, CA) are given in Table 1. Reverse transcription was performed at $95^{\circ} \mathrm{C}$ for $30 \mathrm{~s}$ and circulated at $95^{\circ} \mathrm{C}$ for $40 \mathrm{~s}$, then at $60^{\circ} \mathrm{C}$ for $34 \mathrm{~s}$, and then at $95^{\circ} \mathrm{C}$ for $15 \mathrm{~s}$.

2.9. Determination of TNF- $\alpha I L-6, I L-10, I F N-\gamma$, and $M P O$ Levels in Serum. The levels of TNF- $\alpha$, IL-6, IL-10, IFN- $\gamma$ and MPO were detected by ELISA kit in strict accordance with kit operation instructions. All reagents were purchased from Sigma-Aldrich.

2.10. Statistical Analysis. Data were shown as mean \pm SD. Comparisons between groups of all measured parameters were performed using the one-way ANOVA test and $t$-test. All data were analyzed using SPSS 18 statistical software. $P<0.05$ was considered statistically significant.

\section{Results}

3.1. Effect of Curcumin on Renal Dysfunction. The level of $\mathrm{Cr}$ and BUN was strikingly increased in the RIRI group compared with sham operation $(P<0.01)$ (Figure 1(a)). In this study, significant differences in serum $\mathrm{Cr}$ concentrations were observed in the groups treated with different doses of curcumin during a 24-hour reperfusion period compared with the Sham group, and curcumin significantly reduced the concentration of serum Cr (Figure 1(a)). In summary, the level of serum $\mathrm{Cr}$ in the RIRI group was higher than those in the sham group $(P<0.01)$, while the concentration of serum $\mathrm{Cr}$ in the high-dose curcumin group was dramatically lower than that in the RIRI group $(P<0.05)$.

As shown in Figure 1(b), the concentration of serum BUN was $19.2 \pm 1.91 \mathrm{mg} / \mathrm{dL}$ in the sham group and $58.24 \pm 5.46 \mathrm{mg} / \mathrm{dL}$ in the RIRI group $(P<0.01)$. Compared with the RIRI group, curcumin at three different doses resulted in a notably lower concentration of serum BUN $(P<0.01)$; in addition, the high-dose curcumin group led to a lower concentration of serum BUN compared with medium and low doses $(P<0.05)$. The above results showed that curcumin pretreatment could reduce renal ischemiareperfusion injury in rats, and the higher the concentration of curcumin, the more obvious the antiinjury effect.

3.1.1. Renal Histopathological Observation and Score. Severe congestion and edema were observed in the renal cortex and medulla pallor in the ischemia-reperfusion group. The medulla of the curcumin group showed mild congestion and edema, while no abnormality was observed in the sham group. Under the light microscope, the renal tubules in the ischemia-reperfusion group were notably dilated, the epithelial cells were swollen, vacuolated, and granular degeneration, the capillaries in the glomerulus were dilated, red blood cells were exudated, and interstitial edema and inflammatory cell infiltration were observed. Compared with the RIRI group, the lesion of the curcumin group was prominently reduced, and the renal tissue structure of the sham group was normal. Paller scores were prominently higher in the RIRI group than in the sham group $(P<0.01)$. Paller scores of the curcumin group were prominently decreased compared with the RIRI group $(P<0.01)$, as given in Table 2.

3.2. Assessment of Oxidative Stress. Oxidative stress was assessed by measuring MDA, SOD, CAT, GPX, and GSH, which are the end products of lipid peroxidation caused by reactive oxygen species. Figure 2(a) shows that the level of MDA in the sham group was $56.96 \pm 4.62 \mathrm{nmol} / \mathrm{kg}$. Compared with the RIRI group, the MDA level in the sham group was prominently increased by $78 \%(P<0.01)$. Compared with the RIRI group, curcumin markedly reduced the level of MDA in renal tissues $(P<0.05)$, but still higher than that of the sham operation group $(P<0.01)$, indicating that the expression level of MDA in the curcumin group was negatively correlated with the dose of curcumin; the higher the intervention concentration of curcumin, the lower the level of MDA. The levels of SOD, CAT, GPx, and GSH in the RIRI group were $\quad 186.34 \pm 8.04 \mathrm{U} / \mathrm{g}, \quad 194.31 \pm 11.47 \mathrm{U} / \mathrm{g}$, $41.94 \pm 3.59 \mathrm{U} / \mathrm{g}$, and $24.97 \pm 4.74 \mathrm{nmol} / \mathrm{g}$, respectively, and markedly lower than that in the sham group $(734.98 \pm 12.97 \mathrm{U} / \mathrm{g}, 1021.73 \pm 20.03 \mathrm{U} / \mathrm{g}, 342.94 \pm 12.05 \mathrm{U} / \mathrm{g}$, and $83.43 \pm 6.03 \mathrm{nmol} / \mathrm{g}$, respectively) $(P<0.05)$. The expression levels of SOD, CAT, GPx, and GSH in the curcumin group were markedly higher than those in the RIRI group in dose-dependent manner $(P<0.05)$, indicating that the higher the intervention concentration of curcumin was, the higher the expression level was, as shown in Figure 2. The experimental results revealed that the ischemia-reperfusion injury can produce oxidative stress in rat kidneys, and the antioxidant activity of curcumin could prevent a series of oxidative reactions. Our results also suggested that RIRI caused a significant reduction of FRAP in renal tissue compared to the sham group $(P<0.01)$. Although three different doses of curcumin increased FRAP levels, this change was only significant in the high-dose curcumin group compared with the RIRI group $(P<0.05)$.

3.3. Effect of Curcumin on TNF- $\alpha, I L-6$, and IL- 8 mRNA Level. As given in Table 3, TNF- $\alpha$, IL-6, and IL-8 mRNA levels in the RIRI group were markedly higher than those in the sham group $(P<0.05)$. However, curcumin pretreatment dramatically reduced the levels of TNF- $\alpha$ and IL- 8 than the RIRI group $(P<0.05)$.

3.4. Effects of Curcumin on Anti-Inflammatory Effects. The contents of TNF- $\alpha$, IL- 6 , IL-10, IFN- $\gamma$, and MPO in serum of the RIRI group were dramatically higher than those of the sham group $(P<0.05)$. The expression level of IL-10 in serum of the curcumin group was dramatically higher in a dose-dependent manner than that of the RIRI group $(P<0.05)$; the higher the intervention concentration of curcumin, the higher the expression level of IL-10. The expression levels of TNF- $\alpha$, IFN- $\gamma$, IL-6, and MPO in the curcumin group were memorably lower than those in the RIRI group $(P<0.05)$, indicating that curcumin 
TABle 1: Real-time quantitative PCR primer.

\begin{tabular}{lcc}
\hline Gene & Sense strand sequence & Antisense strand sequence \\
\hline TNF- $\alpha$ & AACACGAGTGACAAGCCCGTAG & GTATCACCAGTTGGTTCTCTTTGA \\
IL-6 & AGGTTCCATGTGCAAGTGTCT & GACAGCCCTGGTCAAAGGTT \\
IL- 8 & CTGCAAGAGACTTCCATCCAG & AGTGGTATAGACAGGTCTGTTGG \\
$\beta$-Actin & AGAGGGAAATCGTGCGTGAC & CAATAGTGATGACCTGGCCGT \\
\hline
\end{tabular}



(a)

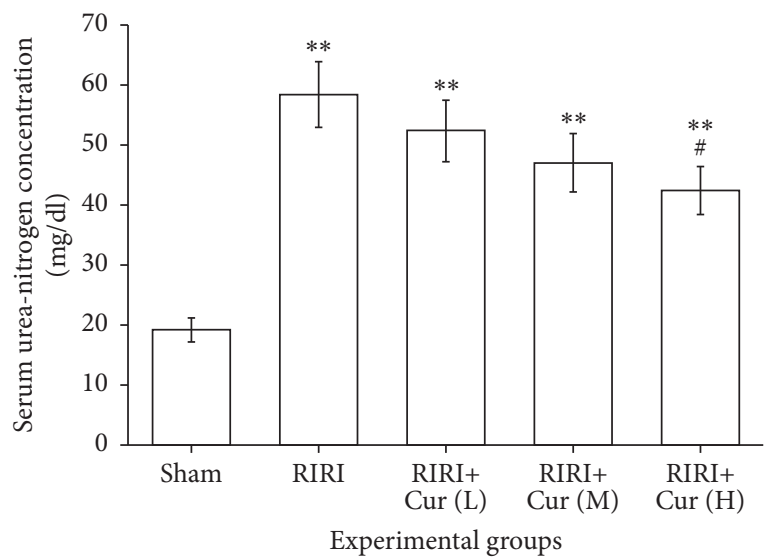

(b)

Figure 1: (a) The concentration of serum $\mathrm{Cr}$ in rats in the RIRI group, sham group, curcumin group L, M, and H, and renal ischemiareperfusion group. (b) The concentration of serum BUN in rats in the RIRI group, sham group, curcumin group L, M, and $\mathrm{H}$, and renal ischemia-reperfusion group $\left({ }^{* *} P<0.01\right.$, vs. the sham group. ${ }^{\#} P<0.05$, vs. the RIRI group). L, low; $M$, medium; $\mathrm{H}$, high.

TABLE 2: Comparison of Paller scores in rats.

\begin{tabular}{lcc}
\hline Group & $n$ & Paller score \\
\hline Sham group & 10 & $103.29 \pm 16.38$ \\
RIRI group & 10 & $509.23 \pm 30.07$ \\
Low-dose curcumin group & 10 & $430.97 \pm 25.13$ \\
Medium-dose curcumin group & 10 & $397.58 \pm 20.75$ \\
High-dose curcumin group & 10 & $351.34 \pm 17.59$ \\
\hline
\end{tabular}

pretreatment decreased the secretion of TNF- $\alpha$, IFN- $\gamma$, IL- 6 , and MPO (Figure 3), and the expression levels of TNF- $\alpha$, IFN- $\gamma$, IL-6, and MPO in the curcumin group were negatively correlated with the dose of curcumin; the higher the intervention concentration of curcumin was, the lower the expression level was, as shown in Figure 3. The results indicated that ischemia-reperfusion injury could lead to inflammatory injury of renal tissue, and the anti-inflammatory effect of curcumin could prevent this pathological process.

3.5. Effects of Curcumin against Apoptosis. The apoptosis number of the RIRI group was $18.87 \pm 1.94$, which was memorably higher than that of the sham group $(7.72 \pm 2.15)$ $(P<0.05)$. The number of apoptotic cells in the curcumin group was memorably lower than that in the RIRI group $(P<0.05)$, suggesting that the apoptosis number of cells in the curcumin group was negatively correlated with the dose of curcumin; the higher the intervention concentration of curcumin, the fewer apoptotic cells. The expression level of caspase-3 in the RIRI group was $61.96 \pm 8.69 \mathrm{ng} / \mathrm{g}$, which was memorably higher than that in the sham group
$(15.27 \pm 2.48 \mathrm{ng} / \mathrm{g})(P<0.05)$. The expression level of caspase-3 in the curcumin group was observably lower than that in the RIRI group $(P<0.05)$, indicating that the expression level of caspase- 3 in the curcumin group was negatively correlated with the dose of curcumin; the higher the intervention concentration of curcumin, the lower the expression level, as shown in Figure 4. The results showed that curcumin alleviated renal ischemia-reperfusion injury via exerting the antiapoptosis effect.

\section{Discussion}

Ischemia-reperfusion injury leads to the deterioration of the function of the transplanted kidney after the restoration of blood supply [19]. RIRI is the main cause of acute kidney injury after partial nephrectomy and kidney transplantation [20], which is closely related to morbidity and mortality [21]. Therefore, effective preventive and therapeutic measures are necessary to reduce renal ischemia-reperfusion injury. Inflammation is an important pathophysiological mechanism of RIRI. RIRI can induce inflammatory cell aggregation, release inflammatory factors (such as TNF- $\alpha$, IL-6 and IL-8), and increase adhesion molecules, which together stimulate inflammatory cascade, leading to organ damage [22]. After renal ischemia, renal cell ischemic necrosis occurs, and then, restored perfusion can cause secondary damage to the kidney [23]. When renal function injury reaches a certain degree, it can exacerbate the level of BUN and $\mathrm{Cr}$ [5].

Current studies on the prevention and treatment of RIRI mainly focus on antioxidants and antiapoptotic drugs 




Experimental groups

(a)

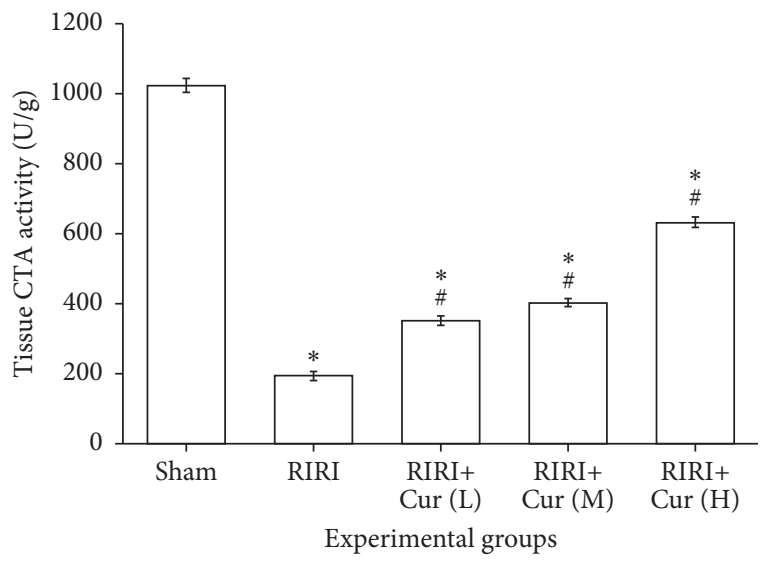

(c)

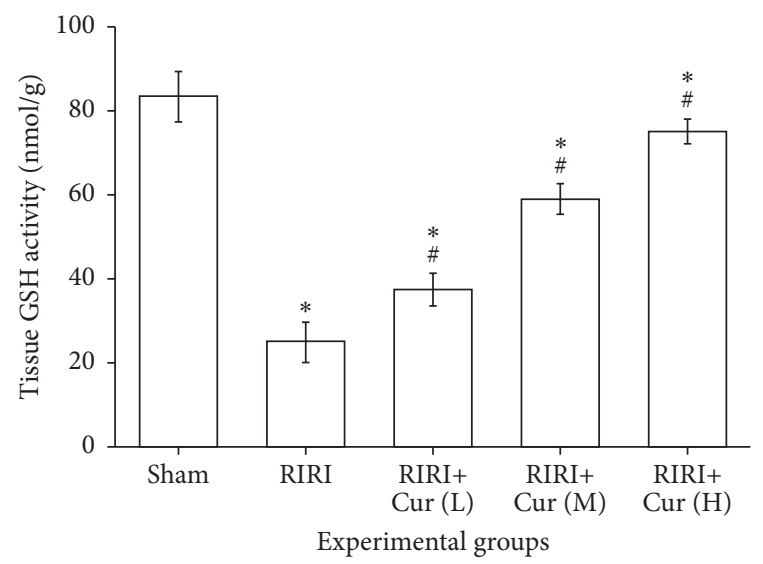

(e)

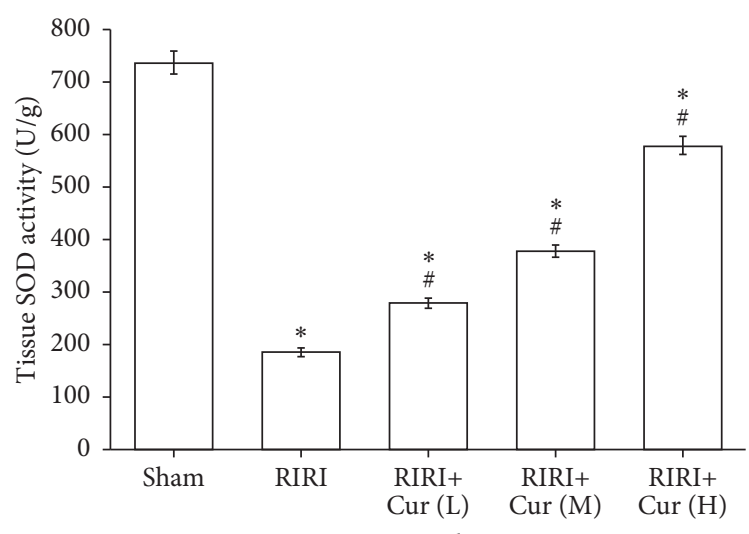

Experimental groups

(b)

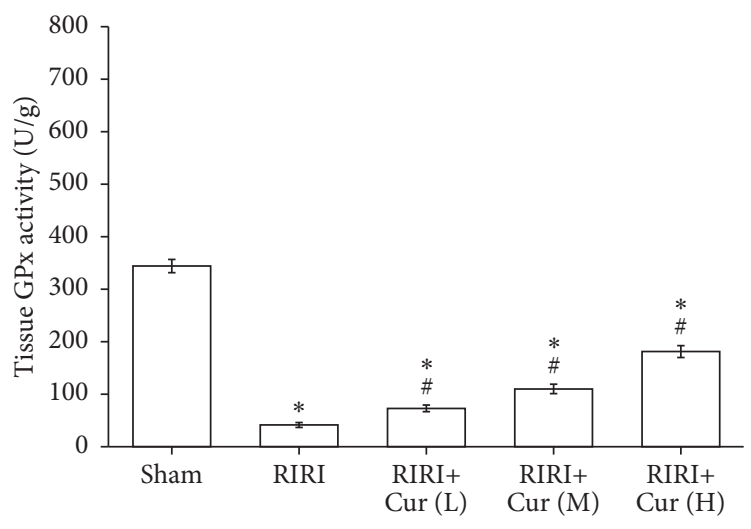

Experimental groups

(d)

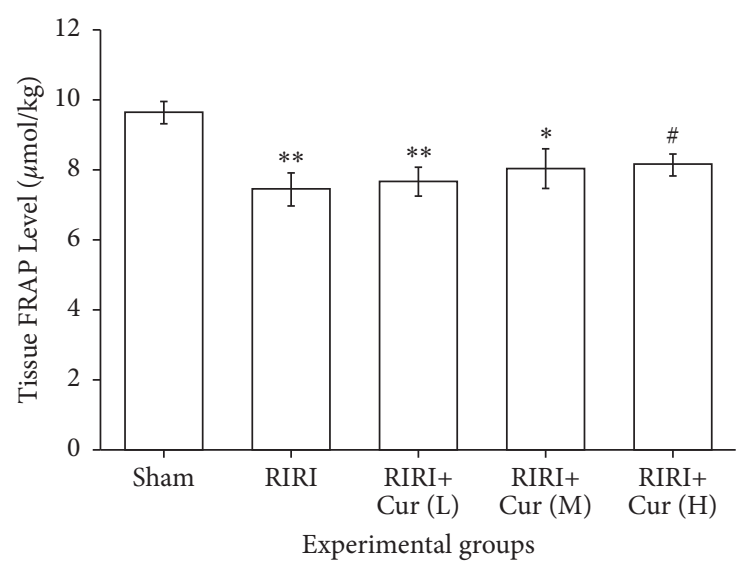

(f)

Figure 2: Protective effect of curcumin on ischemia-reperfusion injury in rats. The levels of MDA (a), SOD (b), CAT (c), GPX (d), GSH (e), and FRAP (f) in renal tissues are detected. ${ }^{*} P<0.05$, vs. the sham group. ${ }^{\#} P<0.05$, vs. the RIRI group.

TABLE 3: Effects of curcumin pretreatment on TNF- $\alpha$, IL-6, and IL-8 mRNA levels in the kidney after renal ischemia-reperfusion injury.

\begin{tabular}{lccc}
\hline Group & TNF- $\alpha$ (mRNA) & IL-6 (mRNA) & IL-8 (mRNA) \\
\hline Sham group & $1.07 \pm 0.19^{* *}$ & $1.14 \pm 0.21^{* *}$ & $0.96 \pm 0.118^{* *}$ \\
RIRI group & $12.36 \pm 1.85$ & $5.09 \pm 0.37$ & $7.63 \pm 0.82$ \\
Low-dose curcumin group & $6.53 \pm 0.99^{*}$ & $2.07 \pm 0.52^{*}$ & $3.77 \pm 0.46^{*}$ \\
Medium-dose curcumin group & $5.87 \pm 0.43^{*}$ & $1.76 \pm 0.47^{*}$ & $2.49 \pm 0.38^{*}$ \\
High-dose curcumin group & $4.74 \pm 0.34^{*}$ & $1.48 \pm 0.39^{*}$ & $2.01 \pm 0.17^{*}$ \\
\hline
\end{tabular}

${ }^{*} P<0.05,{ }^{* *} P<0.01$, vs. the RIRI group. 


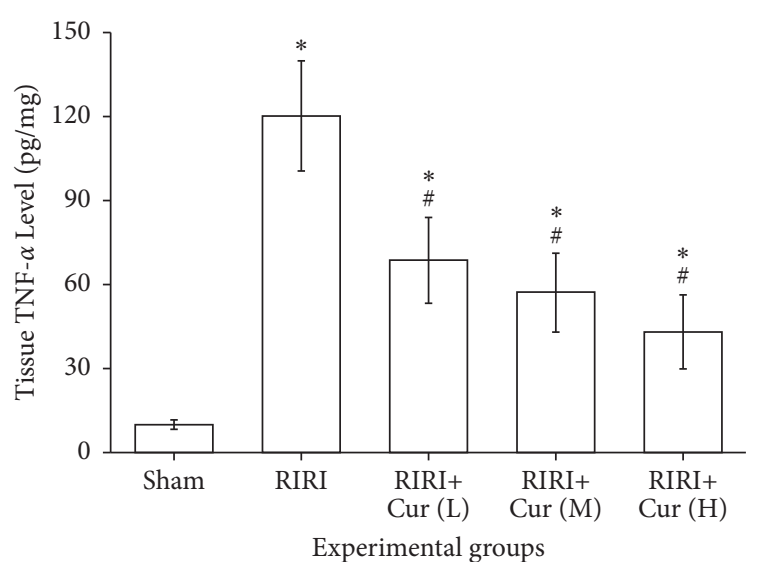

(a)

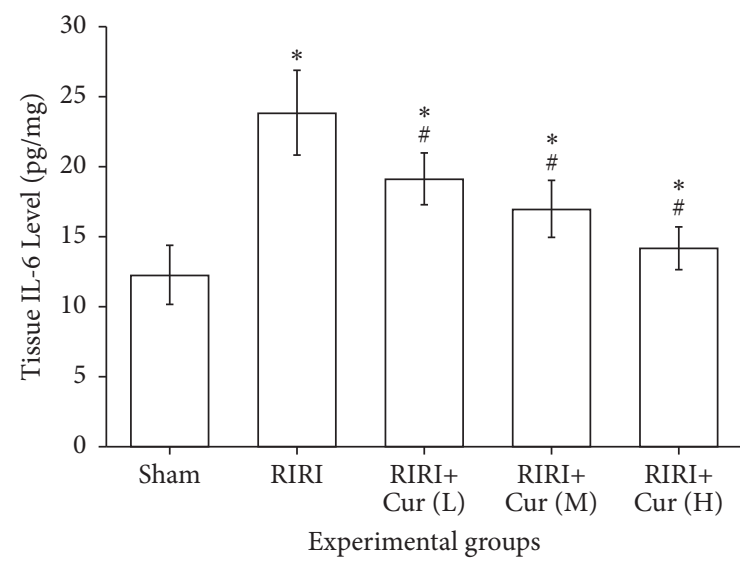

(c)



(b)

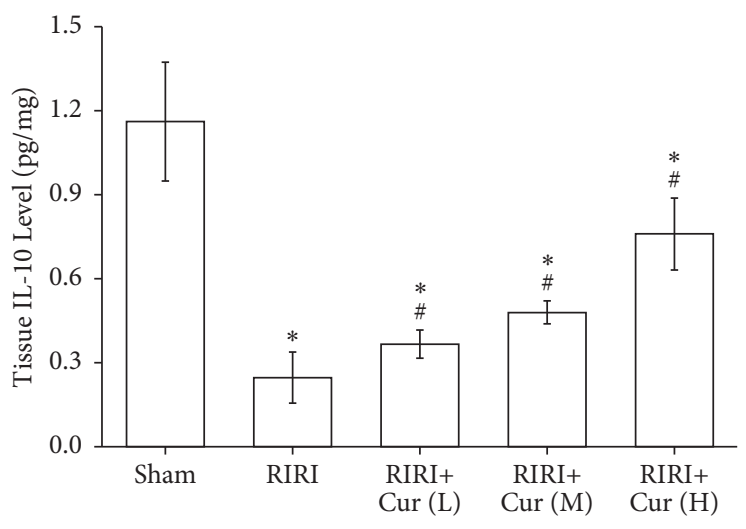

Experimental groups

(d)

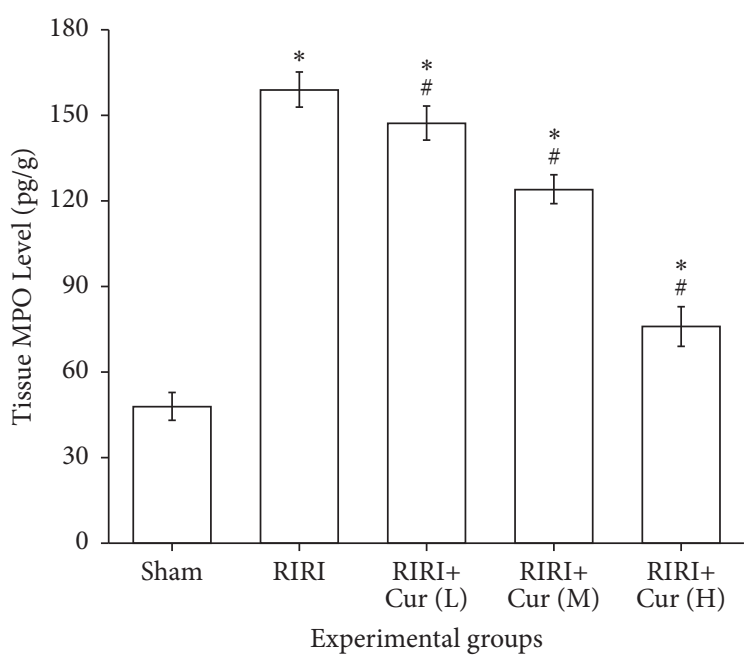

(e)

FIGURE 3: Effect of curcumin pretreatment on expression of TNF- $\alpha$, IFN- $\gamma$, IL-6, IL-10, and MPO in the kidney after renal ischemiareperfusion injury.

[24]. Curcumin is an extract from the rhizome of turmeric, which is an orange knot powder and is widely used as pigment, food additive, and condiment [25]. Previous studies have shown that curcumin has antioxidant [26], anti-inflammatory [27], immunomodulatory [28], and antimicrobial effects [29]. At the same time, curcumin has little toxic and side effects in rodents and humans, and no obvious adverse reactions have been observed even at high doses [30]. Therefore, curcumin has broad medicinal prospects.

The results of this study showed that serum Cr and UBN were highly elevated in the RIRI group, possibly due to a 


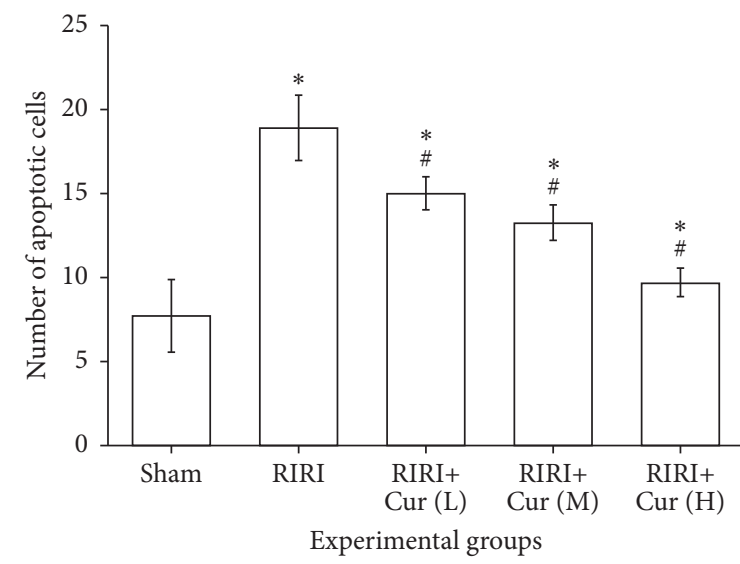

(a)



(b)

FIGURE 4: Effect of curcumin pretreatment on apoptosis of ischemia-reperfusion injury rats vs. the sham group, ${ }^{\#} P<0.05$, and vs. the RIRI group, ${ }^{*} P<0.05$.

sharp decrease in glomerular filtration rate (GFR) and significant damage to renal tubule function in the RIRI group, and the application of curcumin resulted in partial recovery of renal function and reduced oxidative stress induced by RIRI. The content of BUN and $\mathrm{Cr}$ in the RIRI group was higher than those in the sham group, indicating that renal function was obviously damaged. After curcumin treatment, serum BUN and Cr decreased signally, indicating that curcumin has a protective effect on renal function, which is consistent with the findings reported in the literature [6].

When RIRI occurs, the mitochondria of kidney cells are also damaged, leading to decreased activities of SOD and GSH and being unable to effectively remove oxygen free radicals in the body, resulting in the disorder of the body's redox system and aggravating body damage. Curcumin has antioxidant effects and can regulate the activities of GSR, CAT, and SOD in free radicals, thus playing an antiinjury role. Endothelial cell dysfunction during RIRI can produce many inflammatory mediators and release a variety of inflammatory transmitters and adhesion molecules, leading chemotactic neutrophils and inflammatory cells to adhere to vascular endothelial or enter cells. At the same time, neutrophils and other inflammatory cells themselves can release chemotactic substances, which act on the kidney and cause renal ischemia-reperfusion, further aggravating kidney injury. In our study, ischemia/reperfusion resulted in an increased level of MDA and a reduced level of FRAP in the renal tissues of the RIRI group, which was consistent with the previous studies [31]. The content of SOD in the RIRI group was lower than that in the sham group, while the content of MDA was higher than that in the sham group, indicating that after renal ischemia and reperfusion, the body showed obvious lipid peroxidation reaction and weakened antioxidant capacity. Compared with the RIRI group, the content of SOD significantly increased and the content of MDA decreased in the curcumin group, indicating that curcumin treatment can reduce the content of oxygen free radical and inhibit biofilm lipid peroxidation caused by oxygen free radical accumulation, thus enhancing the antioxidant capacity of the kidney and protecting renal ischemia-reperfusion injury [32].

The limitation of this study is that there is no further study on the downstream signaling pathway in which curcumin plays a role. In future studies, we will apply fluorescently labeled curcumin to explore the way in which it acts on cells, such as cell surface receptors or endocytosis.

In summary, curcumin plays a protective role in renal function by preventing cell damage and inhibiting cellular oxidative stress, inflammatory response, and apoptosis, but the specific protective and repair mechanism needs further exploration.

\section{Data Availability}

The data used to support the findings of this study are available from the corresponding author upon request.

\section{Conflicts of Interest}

The authors declare that they have no conflicts of interest.

\section{References}

[1] H. K. Eltzschig and T. Eckle, "Ischemia and reperfusion-from mechanism to translation," Nature Medicine, vol. 17, no. 11, pp. 1391-1401, 2011.

[2] D. P. Basile, D. Donohoe, K. Roethe, and J. L. Osborn, "Renal ischemic injury results in permanent damage to peritubular capillaries and influences long-term function," American Journal of Physiology-Renal Physiology, vol. 281, no. 5, pp. F887-F899, 2001.

[3] H. Zhao, A. Alam, A. P. Soo, A. J. T. George, and D. Ma, "Ischemia-reperfusion injury reduces long term renal graft survival: mechanism and beyond," EBioMedicine, vol. 28, pp. 31-42, 2018.

[4] M. Malek and M. Nematbakhsh, "Renal ischemia/reperfusion injury; from pathophysiology to treatment," Journal of Renal Injury Prevention, vol. 4, no. 2, pp. 20-27, 2015. 
[5] L.-j. Liu, J.-j. Yu, and X.-1. Xu, "Kappa-opioid receptor agonist $\mathrm{U} 50448 \mathrm{H}$ protects against renal ischemia-reperfusion injury in rats via activating the PI3K/Akt signaling pathway," Acta Pharmacologica Sinica, vol. 39, no. 1, pp. 97-106, 2018.

[6] J.-F. Teng, K. Wang, Z.-M. Jia et al., "Lentivirus-Mediated silencing of src homology 2 domain-containing protein tyrosine phosphatase 2 inhibits release of inflammatory cytokines and apoptosis in renal tubular epithelial cells via inhibition of the TLR4/NF-kB pathway in renal ischemiareperfusion injury," Kidney and Blood Pressure Research, vol. 43, no. 4, pp. 1084-1103, 2018.

[7] L. Huang, C. Chen, X. Zhang et al., "Neuroprotective effect of curcumin against cerebral ischemia-reperfusion via mediating autophagy and inflammation," Journal of Molecular Neuroscience, vol. 64, no. 1, pp. 129-139, 2018.

[8] X. Wang, W. Wang, J.-Z. Wang, C. Yang, and C.-Z. Liang, "Effect of apigenin on apoptosis induced by renal ischemia/ reperfusion injury in vivo and in vitro," Renal Failure, vol. 40, no. 1, pp. 498-505, 2018.

[9] J. Wang, W. Lu, L. Chen et al., "Serine 707 of APPL1 is critical for the synaptic NMDA receptor-mediated Akt phosphorylation signaling pathway," Neuroscience Bulletin, vol. 32, no. 4, pp. 323-330, 2016.

[10] F. Liu, W. Ni, J. Zhang, G. Wang, F. Li, and W. Ren, “Administration of curcumin protects kidney tubules against renal ischemia-reperfusion injury (RIRI) by modulating nitric oxide (NO) signaling pathway," Cellular Physiology and Biochemistry, vol. 44, no. 1, pp. 401-411, 2017.

[11] A. S. Awad and A. A. El-Sharif, "Curcumin immune-mediated and anti-apoptotic mechanisms protect against renal ischemia/reperfusion and distant organ induced injuries," International Immunopharmacology, vol. 11, no. 8, pp. 992-996, 2011.

[12] F. H. Liu, W. J. Ni, G. K. Wang, and J. J. Zhang, "Protective role of curcumin on renal ischemia reperfusion injury via attenuating the inflammatory mediators and Caspase-3," Cellular and Molecular Biology (Noisy-Le-Grand, France), vol. 62, no. 11, pp. 95-99, 2016.

[13] C. Nguyen-Ngo, J. C. Willcox, and M. Lappas, "Anti-inflammatory effects of phenolic acids punicalagin and curcumin in human placenta and adipose tissue," Placenta, vol. 100, pp. 1-12, 2020.

[14] S. M. S. Moosavi, S. C. Ashtiyani, and S. Hosseinkhani, "L -carnitine improves oxidative stress and suppressed energy metabolism but not renal dysfunction following release of acute unilateral ureteral obstruction in rat," Neurourology and Urodynamics, vol. 30, no. 3, pp. 480-487, 2011.

[15] S. M. S. Moosavi, S. C. Ashtiyani, S. Hosseinkhani, and M. Shirazi, "Comparison of the effects of l-carnitine and $\alpha$-tocopherol on acute ureteral obstruction-induced renal oxidative imbalance and altered energy metabolism in rats," Urological Research, vol. 38, no. 3, pp. 187-194, 2010.

[16] S. Nezamoleslami, M. Sheibani, F. Jahanshahi, F. Mumtaz, A. Abbasi, and A. R. Dehpour, "Protective effect of dapsone against renal ischemia-reperfusion injury in rat," Immunopharmacology and Immunotoxicology, vol. 42, no. 3, pp. 272-279, 2020.

[17] M. Jafarey, S. Changizi Ashtiyani, and H. Najafi, "Calcium dobesilate for prevention of gentamicin-induced nephrotoxicity in rats," Iranian Journal of Kidney Diseases, vol. 8, no. 1, pp. 46-52, 2014.

[18] A. Kaur, T. Kaur, B. Singh, D. Pathak, H. Singh Buttar, and A. Pal Singh, "Curcumin alleviates ischemia reperfusion-induced acute kidney injury through NMDA receptor antagonism in rats," Renal Failure, vol. 38, no. 9, pp. 1462-1467, 2016.

[19] T. Bergler, U. Hoffmann, E. Bergler et al., "Toll-like receptor 4 in experimental kidney transplantation: early mediator of endogenous danger signals," Nephron. Experimental Nephrology, vol. 121, no. 3-4, pp. e59-70, 2012.

[20] F. Gueler, N. Shushakova, M. Mengel et al., "A novel therapy to attenuate acute kidney injury and ischemic allograft damage after allogenic kidney transplantation in mice," PloS one, vol. 10, no. 1, Article ID e0115709, 2015.

[21] No Authors Listed, "Current world literature," Current Opinion in Nephrology and Hypertension, vol. 19, no. 2, pp. 214-225, 2010.

[22] A. Ahmad, M. A. Sattar, H. A. Rathore et al., "A critical review of pharmacological significance of Hydrogen Sulfide in hypertension," Indian Journal of Pharmacology, vol. 47, no. 3, pp. 243-247, 2015.

[23] M. Ohkita, H. Hayashi, K. Ito et al., "Preventive effects of grape extract on ischemia/reperfusion-induced acute kidney injury in mice," Biological and Pharmaceutical Bulletin, vol. 42, no. 11, pp. 1883-1890, 2019.

[24] E. K. Choi, H. Jung, K. H. Kwak et al., "Inhibition of oxidative stress in renal ischemia-reperfusion injury," Anesthesia \& Analgesia, vol. 124, no. 1, pp. 204-213, 2017.

[25] H. Najafi, S. Changizi Ashtiyani, S. A. Sayedzadeh, Z Mohamadi Yarijani, and S Fakhri, "Therapeutic effects of curcumin on the functional disturbances and oxidative stress induced by renal ischemia/reperfusion in rats," Avicenna Journal of Phytomedicine, vol. 5, no. 6, pp. 576-586, 2015.

[26] S. Abrahams, W. L. Haylett, G. Johnson, J. A. Carr, and S. Bardien, "Antioxidant effects of curcumin in models of neurodegeneration, aging, oxidative and nitrosative stress: a review," Neuroscience, vol. 406, pp. 1-21, 2019.

[27] B. Farhood, K. Mortezaee, N. H. Goradel et al., "Curcumin as an anti-inflammatory agent: implications to radiotherapy and chemotherapy," Journal of Cellular Physiology, vol. 234, no. 5, pp. 5728-5740, 2019.

[28] S. Mohammadian Haftcheshmeh and A. A. Momtazi-Borojeni, "Immunomodulatory therapeutic effects of curcumin in rheumatoid arthritis," Autoimmunity Reviews, vol. 19, no. 8, Article ID 102593, 2020.

[29] S. Sharifi, N. Fathi, M. Y. Memar et al., "Anti-microbial activity of curcumin nanoformulations: new trends and future perspectives," Phytotherapy Research, vol. 34, no. 8, pp. 1926-1946, 2020.

[30] V. Soleimani, A. Sahebkar, and H. Hosseinzadeh, "Turmeric (Curcuma longa) and its major constituent (curcumin) as nontoxic and safe substances: Review," Phytotherapy Research, vol. 32, no. 6, pp. 985-995, 2018.

[31] P. K. Chatterjee, N. S. A. Patel, A. Sivarajah et al., "GW274150, a potent and highly selective inhibitor of iNOS, reduces experimental renal ischemia/reperfusion injury," Kidney International, vol. 63, no. 3, pp. 853-865, 2003.

[32] N. Rogers, M. Stephenson, A. Kitching, J. Horowitz, and P. Coates, "Amelioration of renal ischaemia-reperfusion injury by liposomal delivery of curcumin to renal tubular epithelial and antigen-presenting cells," British Journal of Pharmacology, vol. 166, no. 1, pp. 194-209, 2012. 